\title{
Quality Indicators in Barrett's Esophagus: Time to Change the Status Quo
}

\author{
Samuel Han and Sachin Wani \\ Division of Gastroenterology and Hepatology, University of Colorado Anschutz Medical Center, Aurora, CO, USA
}

The push for high quality care in all fields of medicine highlights the importance of establishing and adhering to quality indicators. In response, several gastrointestinal societies have established quality indicators specific to Barrett's esophagus, which serve to create thresholds for performance while standardizing practice and guiding value-based care. Recent studies, however, have consistently demonstrated the lack of adherence to these quality indicators, particularly in surveillance (appropriate utilization of endoscopy and obtaining biopsies using the Seattle protocol) and endoscopic eradication therapy practices. These findings suggest that innovative interventions are needed to address these shortcomings in order to deliver high quality care to patients with Barrett's esophagus. Clin Endosc 2018;51:344-351

Key Words: Barrett's esophagus; Quality indicators

\section{INTRODUCTION}

Barrett's esophagus (BE) represents a change in the normal epithelium of the esophagus to a columnar-lined intestinal metaplasia. ${ }^{1} \mathrm{BE}$ is believed to progress from non-dysplastic $\mathrm{BE}$ (NDBE) to low-grade dysplasia (LGD), then high-grade dysplasia (HGD) and eventually esophageal adenocarcinoma (EAC). ${ }^{2}$ The increasing incidence of EAC over the past several decades and poor 5-year survival rates have provided an increased impetus to enroll patients in surveillance programs. ${ }^{3}$ These programs focus on the early detection of BE-related neoplasia to reduce the incidence of invasive EAC and ultimately decrease the morbidity and mortality associated with this lethal cancer. To this end, endoscopic eradication

Received: May 30, 2018 Revised: June 13, 2018

Accepted: June 13, 2018

Correspondence: Sachin Wani

Division of Gastroenterology and Hepatology, University of Colorado Anschutz Medical Center, Mail Stop F735, 1635 Aurora Court, Room 2.031, Aurora, CO 80045, USA

Tel: +1-720-848-2786, Fax: +1-820-848-2749, E-mail: sachinwani10@yahoo.com ORCID: https://orcid.org/0000-0002-5150-3623

cc This is an Open Access article distributed under the terms of the Creative Commons Attribution Non-Commercial License (http://creativecommons.org/ licenses/by-nc/3.0) which permits unrestricted non-commercial use, distribution, and reproduction in any medium, provided the original work is properly cited. therapy (EET) has revolutionized the treatment of BE-related neoplasia by not only eradicating neoplasia, but also by maintaining remission and preventing progression to invasive EAC. In light of these advances in technology and therapies, quality indicators become even more important in ensuring that patients receive the highest-value care. Accordingly, the American Gastroenterological Association (AGA), the American Society for Gastrointestinal Endoscopy (ASGE), and the American College of Gastroenterology (ACG) published and endorsed two quality indicator documents that address key quality elements in optimal surveillance and EET practices. ${ }^{4-6}$ Therefore, the aims of this article are to highlight these established and published quality indicators, examine performance in relation to these quality indicators, and address the future prospects for optimized care in BE.

\section{QUALITY INDICATORS}

Defined as the "degree to which health services for individuals and populations increase the likelihood of desired health outcomes and are consistent with current professional knowledge", quality remains paramount in providing the best possible care for each individual patient. ${ }^{7}$ In countries such as the 
United States, reimbursement models continue to shift away from fee-for-service to a model based on quality and outcome measures that prioritizes value-based care; this makes the ability to measure quality a critical component of confirming that patients do in fact receive high-quality care. To this end, quality indicators have a substantial role to play.

Quality indicators help standardize high-quality care by serving as a reference point by which performance can be measured. With regard to BE specifically, reliable measurements of endoscopist performance are needed to improve the value of endoscopy for screening, surveillance, and treatment. ${ }^{8}$ The three main types of quality indicators include structural measures that assess the entire health care environment, process measures that assess the actual delivery of care, and outcome measures that assess the actual results of the care that was given. ${ }^{9}$ The National Quality Forum emphasizes five essential features of a high quality indicator: (1) Is the indicator evidence-based and does it address a priority performance gap? (2) Is the indicator well specified to allow it to be compared across organizations and does it reliably differentiate high and low-quality clinicians? (3) Are the data required for the indicator readily available or easily captured? (4) Can stakeholders use the indicator for both accountability and quality improvement to improve the quality of care? and
(5) Are there other indicators for the same concept? ${ }^{8}$ Overall, there is a need for more outcome measures to improve patient outcomes such as mortality, cancer progression, or endoscopic adverse events. ${ }^{8}$

\section{QUALITY INDICATORS FOR BARRETT'S ESOPHAGUS}

The quality indicators specific to $\mathrm{BE}$ outlined in this review were derived from two main documents: (1) Quality Indicators for the Management of Barrett's Esophagus, Dysplasia, and Esophageal Adenocarcinoma: International Consensus Recommendations from the AGA Symposium; and (2) the quality indicators document for EET in BE by Wani et al. endorsed by the ACG and the ASGE. ${ }^{4-6}$ The AGA document utilized a modified Delphi method including 25 international experts to create a list of quality indicators for multiple aspects of BE. An agreement of at least $80 \%$ was needed for final inclusion of the quality indicator, which included an accompanying assessment of the evidence. ${ }^{4}$ These quality indicators are outlined in Table 1 . The more recent Treatment with Resection and Endoscopic Ablation Techniques for BE (TREAT$\mathrm{BE}$ ) Consortium document endorsed by the ACG and the

Table 1. Quality Indicators for the Treatment and Management of Barrett's Esophagus, Neoplasia, and Early Cancer Based on a Modified Delphi Method from Sharma et al. ${ }^{4}$

\begin{tabular}{|c|c|c|}
\hline Quality indicator & Agreement & Grade of recommendation \\
\hline \multicolumn{3}{|l|}{ Screening, diagnosis, and staging } \\
\hline $\begin{array}{l}\text { For patients in whom BE is suspected, the squamo-columnar junc- } \\
\text { tion, the gastroesophageal junction, and the location of the dia- } \\
\text { phragmatic hiatus (if there is a hiatal hernia present) should be } \\
\text { recorded on each upper endoscopy }\end{array}$ & $\begin{array}{c}87 \% \\
(35 \% \text { strongly agree, } 52 \% \text { agree })\end{array}$ & Weak \\
\hline $\begin{array}{l}\text { If } \mathrm{BE} \text { is suspected on endoscopy, the endoscopist should document } \\
\text { the extent of suspected BE using the Prague criteria }\end{array}$ & $\begin{array}{c}82.6 \% \\
(43.5 \% \text { strongly agree, } 39.1 \% \text { agree })\end{array}$ & Weak \\
\hline \multicolumn{3}{|l|}{ Surveillance } \\
\hline $\begin{array}{l}\text { If systematic surveillance biopsies performed in a patient known to } \\
\text { have BE show no evidence of dysplasia, follow-up surveillance en- } \\
\text { doscopy should be recommended no sooner than } 3 \text { to } 5 \text { years }\end{array}$ & $\begin{array}{c}91.3 \% \\
(17.3 \% \text { strongly agree, } 74 \% \text { agree })\end{array}$ & Weak \\
\hline $\begin{array}{l}\text { If a patient with known BE undergoes surveillance endoscopy, sys- } \\
\text { tematic biopsies should be taken every } 1-2 \mathrm{~cm} \text { from } 4 \text { quadrants } \\
\text { throughout the extent of the endoscopically involved segment }\end{array}$ & $\begin{array}{c}95.7 \% \\
(52.2 \% \text { strongly agree, } 43.5 \% \text { agree })\end{array}$ & Strong \\
\hline \multicolumn{3}{|l|}{ Treatment and management } \\
\hline $\begin{array}{l}\text { In patients with dysplastic BE or early esophageal adenocarcinoma, a } \\
\text { diagnostic endoscopic resection should be performed on any raised } \\
\text { or suspicious areas }\end{array}$ & $\begin{array}{c}95.6 \% \\
(65.2 \% \text { strongly agree, } 30.5 \% \text { agree })\end{array}$ & Strong \\
\hline $\begin{array}{l}\text { In patients with } \mathrm{BE} \text {-associated neoplasia, the goal of endoscopic } \\
\text { treatment should be the complete eradication of the BE segment in } \\
\text { addition to any dysplastic lesions }\end{array}$ & $\begin{array}{c}100 \% \\
(65.2 \% \text { strongly agree, } 34.8 \% \text { agree })\end{array}$ & Strong \\
\hline
\end{tabular}

BE, Barrett's esophagus. Adapted with permission from Sharma et al. ${ }^{4}$ 
ASGE developed quality indicators using the RAND/University of California, Los Angeles Appropriateness Methodology (RAM) system. ${ }^{5,6}$ Nineteen international experts participated in the selection process, with the final inclusion of 14 quality indicators organized into pre-procedure, intra-procedure, and post-procedure sections. The RAM system enabled the benefits and harms of endoscopic treatments to be weighed and established thresholds for performance in the outlined quality indicators. The composite of these quality indicators is presented in Table 2.

\section{Screening, diagnosis, and staging}

For patients in whom BE is being considered, the squamo-columnar junction, the gastroesophageal junction, and the location of the diaphragmatic hiatus (if there is a hiatal hernia present) should be recorded on each upper endoscopy

This consensus recommendation from the AGA illustrates the importance of the proper documentation of major land- marks. ${ }^{4}$ Detection and documentation of these landmarks are associated with a high inter- and intra-observer agreement, allowing for reliable reproducibility. ${ }^{4,10}$ While endoscopists often photo-document these landmarks, the location of these landmarks, expressed as the distance $(\mathrm{cm})$ from the incisors, should be included in the endoscopy report to standardize the relative location of the columnar-lined epithelium for each patient. ${ }^{5}$ A recent study utilizing the Danish Pathology Registry found that in 300 patients with $\mathrm{BE}$, documentation of the gastroesophageal junction and the squamo-columnar junction was performed by only $23 \%$ and $26 \%$ of endoscopists, respectively. ${ }^{11}$ It should be noted that studies have yet to establish improvement in patient outcomes with proper documentation of landmarks.

If $B E$ is suspected on endoscopy, the endoscopist should document the extent of suspected BE using the Prague criteria

The Prague C \& M criteria allows for the documentation of

Table 2. Quality Indicators for Endoscopic Eradication Therapy Based on the RAND/UCLA Appropriateness Methodology from Wani et al. ${ }^{5,6}$

\begin{tabular}{|c|c|c|}
\hline Quality indicator & Threshold & Process or outcome measure \\
\hline \multicolumn{3}{|l|}{ Pre-procedure } \\
\hline $\begin{array}{l}\text { For patients in whom a diagnosis of dysplasia has been made, the rate at which the } \\
\text { reading is made by a GI pathologist or confirmed by a second pathologist before } \\
\text { EET is initiated }\end{array}$ & $\begin{array}{c}90 \% \\
(75,100)\end{array}$ & Process \\
\hline $\begin{array}{l}\text { If EET is performed, HD-WLE and expertise in mucosal ablation and EMR tech- } \\
\text { niques should be available }\end{array}$ & N/A & Process \\
\hline $\begin{array}{l}\text { The rate at which documentation of a discussion of the risks, benefits, and alterna- } \\
\text { tives to EET is obtained from the patient before a course of treatment is initiated }\end{array}$ & $\begin{array}{c}99 \% \\
(85,100)\end{array}$ & Process \\
\hline \multicolumn{3}{|l|}{ Intra-procedure } \\
\hline $\begin{array}{l}\text { The rate at which the landmarks and length of Barrett's esophagus are documented } \\
\text { (e.g., Prague grading system) in patients with Barrett's esophagus before EET }\end{array}$ & $\begin{array}{c}90 \% \\
(75,100)\end{array}$ & Process \\
\hline $\begin{array}{l}\text { The rate at which the presence or absence of visible lesions is reported (e.g., Paris } \\
\text { classification) in patients with Barrett's esophagus referred for EET }\end{array}$ & $\begin{array}{c}90 \% \\
(60,100)\end{array}$ & Process \\
\hline The rate at which the Barrett's esophagus segment is inspected using HD-WLE & $\begin{array}{c}95 \% \\
(0,100)\end{array}$ & Process \\
\hline $\begin{array}{l}\text { The rate at which CE-IM is achieved by } 18 \text { months in patients with Barrett's-relat- } \\
\text { ed dysplasia and intramucosal cancer referred for EET }\end{array}$ & $\begin{array}{c}70 \% \\
(50,80)\end{array}$ & Outcome \\
\hline \multicolumn{3}{|l|}{ Post-procedure } \\
\hline $\begin{array}{l}\text { Among patients who achieve CE-IM, the rate at which a recommendation for en- } \\
\text { doscopic surveillance at a defined interval is documented }\end{array}$ & $\begin{array}{c}90 \% \\
(50,100)\end{array}$ & Process \\
\hline $\begin{array}{l}\text { During endoscopic surveillance after EET, the rate at which biopsies of any visible } \\
\text { mucosal abnormalities are performed }\end{array}$ & $\begin{array}{c}95 \% \\
(50,100)\end{array}$ & Process \\
\hline The rate at which an anti-reflux regimen is recommended after EET & $\begin{array}{c}90 \% \\
(50,100)\end{array}$ & Process \\
\hline $\begin{array}{l}\text { The rate at which adverse events are tracked and documented in individuals after } \\
\text { EET }\end{array}$ & $\begin{array}{c}90 \% \\
(50,100)\end{array}$ & Process \\
\hline
\end{tabular}

GI, gastrointestinal; EET, endoscopic eradication therapy; HD-WLE, high-definition white light endoscopy; EMR, endoscopic mucosal resection; N/A, not available; CE-IM, complete eradication of intestinal metaplasia. Reprinted with permission from Wani et al. ${ }^{6}$ 
the circumferential and maximal extent of the visualized BE segment, thereby standardizing the reporting of the length of $\mathrm{BE}$ in each patient. ${ }^{10}$ The reliability of these criteria has been validated in multiple studies involving experts and trainees, as well as in endoscopists from Western and Asian countries. The importance of documenting the length of BE via the Prague Criteria is particularly significant in EET, where endoscopists must standardize the location and extent of therapy. In terms of adherence, the same study from Denmark mentioned above also found that only $34 \%$ of endoscopy reports included the Prague classification. ${ }^{11}$ Similarly, in a recent study, Han et al. showed that the overall Prague criteria adherence rate in patients with histologically confirmed BE was only $41.8 \%$ at a tertiary academic medical center in the US. ${ }^{12}$ Education regarding the Prague criteria may play a significant role in adherence, as demonstrated by Ooi et al. who implemented a teaching program that resulted in a $93 \%$ adherence rate compared to a $16 \%$ adherence rate $(p<0.001)$ in a group of endoscopists who did not receive the educational intervention. ${ }^{13}$ Similar to landmark documentation, however, use of the Prague criteria has not been shown to improve patient outcomes, which represents a potential area for future investigation.

\section{The rate at which the presence or absence of visible lesions is re- ported in patients with BE referred for EET}

Visible lesions create cause for concern due to their potential to contain neoplasia. The TREAT-BE consortium recommends the use of the Paris classification to uniformly grade visible lesions. Put simply, the Paris classification separates protruded lesions from flat lesions. ${ }^{5}$ Non-flat lesions include (1) 0 -Ip (pedunculated) and (2) 0-Is (sessile). Flat lesions consist of 0-IIa (superficially elevated), 0-IIb (flat), 0-IIc (superficially depressed), and 0-III (excavated). Lesions with the 0-Is, 0-IIc, and 0 -III classification are more likely to carry invasive cancer, whereas 0-IIa and 0-IIb lesions are unlikely to carry invasive cancer. However, there is currently no evidence that demonstrates an improvement in patient outcomes with the use of the Paris classification.

\section{The rate at which the $B E$ segment is inspected using high-defini- tion white light endoscopy}

High-definition white light endoscopy (HD-WLE) enables the procurement of a higher-resolution image that can provide a clearer view of the mucosa affected by $\mathrm{BE}$ and has become the standard of care in the primary evaluation of patients with BE. ${ }^{1}$ While no randomized controlled trial has directly compared HD-WLE with standard-definition white light endoscopy, data from at least four high-quality observational studies comparing random biopsies using HD-WLE with targeted biopsies using narrow band imaging, including that by Sharma et al., indicate that HD-WLE has a higher sensitivity than standard-definition white light endoscopy in finding BE-related neoplasia. ${ }^{6,14}$

\section{Surveillance}

If systematic surveillance biopsies performed in a patient known to have BE show no evidence of dysplasia, follow-up surveillance endoscopy should be recommended no sooner than 3 to 5 years

While this quality indicator received a weak recommendation from the AGA with a low quality of supporting evidence, its importance derives from the need to reduce unnecessary and frequent endoscopy that adds minimal benefit to patients. As Shaheen et al. recently pointed out, a minimalist approach to endoscopy is crucial to increase the yield of clinically relevant information for the patient while decreasing the costs and risks associated with endoscopy. ${ }^{15} \mathrm{~A}$ recent study by Wani et al. utilizing the GI Quality Improvement Consortium (GIQuIC) registry, a national data repository of endoscopy quality measures from the US, found that of the 25,945 patients with NDBE, $26.9 \%$ were recommended surveillance at 1-2 years, which is much sooner than the minimum 3-year interval. ${ }^{16}$ Similarly, Tavakkoli et al. showed in a single-center study that only $15.9 \%$ of patients with NDBE underwent appropriate surveillance, with $37.9 \%$ receiving over-surveillance. ${ }^{17}$ Notably, they found that the presence of a primary care physician in their practice decreased the risk of over-surveillance, which represents a potential way to improve appropriate surveillance in this patient population. Given that NDBE is associated with a very low risk of progression to EAC, application of this quality indicator may result in substantial cost savings while adding minimal risk. ${ }^{18}$

If a patient with known BE undergoes surveillance endoscopy, systematic biopsies should be taken every 1-2 cm from 4 quadrants throughout the extent of the endoscopically involved segment

The Seattle protocol, consisting of systematic 4-quadrant biopsies every $2 \mathrm{~cm}$ throughout the length of $\mathrm{BE}$, was recommended by the AGA for performing surveillance. ${ }^{4}$ In support of this, Abrams et al. evaluated 2,245 surveillance endoscopies and found that the odds of detecting dysplasia decreased with nonadherence to a systematic biopsy protocol (odds ratio [OR], 0.53 ; $95 \%$ confidence interval [CI], $0.35-0.82$ ), highlighting the clinical importance of standardized tissue sampling. ${ }^{19}$ Several studies have examined the adherence by endoscopists to performing the Seattle protocol for surveillance. In a single-center retrospective study examining 397 patients who received an upper endoscopy for BE, Westerveld et al. found adherence to 
the Seattle protocol to be only $24.1 \%{ }^{20}$ Additionally, endoscopists with a longer time in practice had a lower likelihood of performing the Seattle protocol (OR, 0.91; 95\% CI, 0.85-0.97, $p<0.01)$. In a prospective study, Ooi et al. incorporated a training program to teach concepts such as the Seattle protocol and found that after the training program, endoscopists had a $77 \%$ adherence rate to the Seattle protocol, which was significantly higher than the cohort of endoscopists who did not participate in the training program $(10 \%, p<0.001) .{ }^{13}$ Lastly, a recent study utilizing the GIQuIC registry assessed 58,709 upper endoscopies in the United States and found a Seattle protocol adherence rate of between $73 \%-77.5 \%{ }^{21}$ Surprisingly, there was a strong inverse association between $\mathrm{BE}$ length and adherence $(p<0.0001)$, suggesting that patients with the highest risk of dysplasia do not receive proper sampling. Data from this registry also revealed regional variation in adherence rates, with the Northeast region of the US having a 81.4\%-84.8\% adherence rate compared to the Midwest region of the US, which had an adherence rate from $62.5 \%-67.8 \%$, which implies variability in practice patterns by geography. ${ }^{22}$

\section{Treatment}

For patients in whom a diagnosis of dysplasia has been made, the rate at which the reading is made by a gastrointestinal pathologist or confirmed by a second pathologist before EET is initiated

This important quality indicator highlights the substantial interobserver variability in the interpretation of dysplasia by pathologists. In a study by Curvers et al., two expert pathologists reviewed the histology of 147 patients diagnosed with LGD at community practices. ${ }^{23}$ After review, $85 \%$ of the patients were downstaged to either LGD or indefinite for dysplasia. Endoscopic follow-up validated the re-staging as the risk of progression differed significantly between patients with confirmed LGD (13.4\% per patient per year) and downgraded patients ( $0.49 \%$ per patient per year). A meta-analysis by Qumseya et al. confirmed these findings, demonstrating a significantly higher cumulative rate of progression from LGD in studies where an expert gastrointestinal pathologist/ panel of pathologists confirmed the histological diagnosis of LGD. $^{24}$ Therefore, an expert pathologist or group of pathologists should review diagnoses of BE-associated dysplasia to not only provide the best prognostic information, but also to guide treatment and/or surveillance. The TREAT-BE Consortium also advocates for standardization in reporting dysplasia via use of the Vienna classification, as follows: (1) negative for dysplasia, (2) indefinite for dysplasia, (3) LGD, (4) HGD, and (5) invasive neoplasia. ${ }^{5}$
If EET is performed, HD-WLE and expertise in mucosal ablation and endoscopic mucosal resection techniques should be available

This process measure reflects the need for appropriate equipment to detect and treat $\mathrm{BE}$. Although no randomized studies have directly analyzed HD-WLE against standard definition-white light endoscopy, Schölvinck et al. found a significantly lower neoplastic detection rate in community hospitals $(60 \%)$ than in expert centers $(87 \%, p<0.001)$, which attests to the importance of having the necessary resources to provide high-quality care. ${ }^{25}$ As few studies have examined the level of proficiency needed for ablation and endoscopic mucosal resection (EMR), this indicator cannot address the minimum thresholds for competence in these techniques.

The rate at which documentation of a discussion of the risks, benefits, and alternatives to EET is obtained from the patient before a course of treatment is initiated

Detailed and thorough informed consent enables patients to understand the risks and benefits of any therapy and gives them the necessary information to make a decision that is consistent with their beliefs and desires. For BE in particular, important aspects include the risk of progression, goals of EET, potential need for repeat endoscopies, post-procedure treatments such as acid suppression, and surveillance intervals after complete eradication of intestinal metaplasia (CE-IM). Future studies should examine the impact of comprehensive informed consent on patient satisfaction.

The rate at which endoscopic resection (en bloc or piecemeal) should be performed on any visible lesions

As supported by both quality indicator documents and several observational studies, endoscopic resection can improve histologic accuracy and offers therapeutic potential in addition to its diagnostic capabilities. ${ }^{4,5}$ As Moss et al. demonstrated in their prospective study of 75 patients, endoscopic resection resulted in a change in the grading or staging in $48 \%$ of patients, and after a median follow-up period of 31 months, no recurrence occurred at the resection sites in the entire cohort. ${ }^{26}$ Comparing EMR to standard biopsy, Wani et al. showed that the interobserver agreement in the diagnosis of dysplasia was significantly greater for EMR specimens $(n=251)$ than for biopsy specimens $(n=269))^{27}$ Furthermore, in a recent meta-analysis by Wani et al. including 14 studies, a random-effects model demonstrated that EMR resulted in a change in the pathologic diagnosis in 39\% (95\% CI, 34\%-45\%) of all patients. ${ }^{28}$ 
The rate at which complete eradication of neoplasia is achieved by 18 months in patients with BE-related dysplasia or intramucosal cancer referred for EET

The rate at which CE-IM is achieved by 18 months in patients with BE-related dysplasia and intramucosal cancer referred for EET

These two quality indicators speak to the importance of achieving the treatment goal in line with published rates for the CE-IM and neoplasia, of which CE-IM remains the primary treatment goal. As Pech et al. demonstrated in a longterm follow-up study of 349 patients with HGD or EAC, CE of dysplasia (CE-D) occurred in $96.6 \%$ of patients with endoscopic therapy; however, metachronous lesions developed in $21.5 \%$ of patients, suggesting that CE-D alone does not facilitate the ideal endpoint for patients. ${ }^{29}$ Two major randomized controlled trials by Shaheen et al. and Phoa et al. have set the benchmark for the performance of EET in achieving CE-D and CE-IM. ${ }^{30,31}$ In the Ablation of Intestinal Metaplasia dysplasia trial by Shaheen et al. involving patients with LGD and HGD, CE-D and CE-IM was achieved in 90.5\% (for LGD) and $77.4 \%$ of patients, respectively. ${ }^{30}$ Furthermore, patients in the radiofrequency ablation (RFA) group had a lower rate of disease progression ( $3.6 \%$ vs. $16.3 \%, p=0.03$ ) than did the control group. Similarly, the trial by Phoa et al. randomized patients with LGD to either RFA or surveillance and demonstrated a CE-D rate of $92.6 \%$ and CE-IM rate of $88.2 \%{ }^{31}$ Ablation also significantly reduced the risk of progression (1.5\% vs. $26.5 \%$, $p<0.001)$ in these patients. Overall, RFA has been found to have a CE-D rate of $91 \%(95 \% \mathrm{CI}, 87 \%-95 \%)$ and a CE-IM rate of $78 \%$ (95\% CI, 70\%-86\%). ${ }^{6}$ The timeframe of 18 months was chosen to reflect the median number of sessions required to achieve CE-IM in these trials, but no data currently suggest that this timeline improves outcomes such as progression.

\section{Post-treatment}

Among patients who achieve CE-IM, the rate at which a recommendation is documented for endoscopic surveillance at a defined interval

Although CE-IM remains the goal of all endoscopic therapy in patients with dysplastic $\mathrm{BE}$, recurrence remains a serious concern as it can occur in 5\%-39.5\% of patients after CE-IM. ${ }^{5}$ Despite strong agreement on the necessity of surveillance, there is little evidence to guide the establishment of optimal surveillance intervals. Recently, however, Cotton et al. collected data from the United States Radiofrequency Ablation Registry (5,444 patients from 2004-2013) and the United Kingdom National Halo Registry (391 patients from 2007-2015) to build and validate Cox proportional hazard models to predict neoplasia recurrence rates following successful RFA. ${ }^{32}$
These models created three risk groups based on the baseline grade of dysplasia categorized into (1) NDBE or indefinite for dysplasia, (2) LGD, and (3) HGD or intramucosal adenocarcinoma. Patients with LGD had a neoplastic recurrence rate of 2.0, leading to a suggested surveillance endoscopy schedule of 1 and 3 years after CE-IM. For patients with HGD or EAC, a neoplasia recurrence rate of 5.5 was found, leading to a recommended surveillance schedule of $0.25,0.5$, and 1 year after CE-IM and annually thereafter.

The rate at which an anti-reflux regimen is recommended after EET

This statement builds on evidence that improved acid control is associated with improved outcomes after EET such as RFA. In a multicenter study including 278 patients with BE treated with circumferential RFA, van Vilsteren et al. found that an independent predictor for a poor response to treatment included ongoing reflux esophagitis (OR, 37.4; 95\% CI, $3.2,433.2){ }^{33}$ Similarly, Komanduri et al. followed 221 consecutive patients treated with RFA. ${ }^{34}$ In patients who did not achieve CE-IM within three sessions of RFA, the only predictive factor for incomplete response was a reduction in dose or frequency of proton pump inhibitor during EET. Additionally, among incomplete responders, 93.8\% eventually achieved CEIM after acid reduction modifications (primarily fundoplication and alteration in acid suppressive medications) after a mean of 1.1 additional RFA sessions.

\section{The rate at which adverse events are tracked and documented in individuals after EET}

The tracking and documentation of adverse events remain a cornerstone in providing high-quality care to patients with BE. A meta-analysis by Qumseya et al. demonstrated an overall $8.8 \%$ adverse event rate for RFA in patients with $\mathrm{BE} .^{35}$ Adverse events included strictures (5.6\%), bleeding (1\%), and perforation $(0.6 \%)$. The individual rates of these adverse events for each endoscopist should be relayed to each patient to fully disclose the risks of EET.

\section{CONCLUSIONS}

In the changing world of healthcare economics and owing to the call for a shift to value-based care, quality indicators play a significant role in guiding physicians, patients, and payers in the delivery of high-quality care. The two primary documents discussed in this review have established standards for the care of patients with $\mathrm{BE}$, providing a framework for effective assessments of care. ${ }^{4,5}$ Unfortunately, as demonstrated by the discussed studies that include large national registries, adherence to these quality indicators remains poor. 
Although quality indicators may set objective thresholds for performance, the value of care will not improve until physicians adhere to these measures. Therefore, in order to change the status quo, future studies will need to focus on the implementation of these quality indicators while designing interventions to improve adherence. Educational programs appear to be effective in changing behavior, but these programs will need to be assessed on a prospective, multicenter scale. Integrating quality indicators into electronic medical records or incentivizing adherence may also facilitate their incorporation into clinical practice, which, despite its inherent challenges, promises to ultimately improve care in patients with $\mathrm{BE}$.

\section{Conflicts of Interest}

This work was supported by the University of Colorado, Department of Medicine Outstanding Early Scholars Program (SW) and NIH T32DK007038-42 (SH). Consultant - Medtronic, Boston Scientific (SW).

\section{REFERENCES}

1. Fitzgerald RC, di Pietro M, Ragunath K, et al. British Society of Gastroenterology guidelines on the diagnosis and management of Barrett's oesophagus. Gut 2014;63:7-42.

2. Wani S, Falk GW, Post J, et al. Risk factors for progression of lowgrade dysplasia in patients with Barrett's esophagus. Gastroenterology 2011;141:1179-1186, 1186.e1.

3. Thrift AP. Barrett's esophagus and esophageal adenocarcinoma: how common are they really? Dig Dis Sci 2018;63:1988-1996.

4. Sharma P, Katzka DA, Gupta N, et al. Quality indicators for the management of Barrett's esophagus, dysplasia, and esophageal adenocarcinoma: international consensus recommendations from the American Gastroenterological Association Symposium. Gastroenterology 2015;149:1599-1606.

5. Wani S, Muthusamy VR, Shaheen NJ, et al. Development of quality indicators for endoscopic eradication therapies in Barrett's esophagus: the TREAT-BE (Treatment with Resection and Endoscopic Ablation Techniques for Barrett's Esophagus) Consortium. Gastrointest Endosc 2017;86:1-17.e3

6. Wani S, Muthusamy VR, Shaheen NJ, et al. Development of quality indicators for endoscopic eradication therapies in Barrett's esophagus: the TREAT-BE (Treatment With Resection and Endoscopic Ablation Techniques for Barrett's Esophagus) Consortium. Am J Gastroenterol 2017;112:1032-1048.

7. Institute of Medicine (US). Americas health in transition: protecting and improving quality. Washington, D.C.: National Academies Press (US); 1994.

8. Rubenstein JH, Lieberman D, Fennerty B, Gellad ZF. Measuring the quality of Barrett's esophagus management with measures that are high quality. Gastroenterology 2015;149:1298-1301.

9. Rizk MK, Sawhney MS, Cohen J, et al. Quality indicators common to all GI endoscopic procedures. Gastrointest Endosc 2015;81:3-16.

10. Sharma P, Dent J, Armstrong D, et al. The development and validation of an endoscopic grading system for Barrett's esophagus: the Prague C \& M criteria. Gastroenterology 2006;131:1392-1399.

11. Vogt JS, Larsen AC, Sommer T, Ejstrud P. Quality of endoscopic surveillance of Barrett's esophagus. Scand J Gastroenterol 2018;53:256-259.

12. Han S, Yadlapati R, DeLay K, et al. Factors associated with suboptimal adherence to quality indicators in Barrett's esophagus. Gastroenterology 2018;154(6 Suppl 1):S-140.
13. Ooi J, Wilson P, Walker G, et al. Dedicated Barrett's surveillance sessions managed by trained endoscopists improve dysplasia detection rate. Endoscopy 2017;49:524-528.

14. Sharma P, Hawes RH, Bansal A, et al. Standard endoscopy with random biopsies versus narrow band imaging targeted biopsies in Barrett's oesophagus: a prospective, international, randomised controlled trial. Gut 2013;62:15-21.

15. Shaheen NJ, Fennerty MB, Bergman JJ. Less is more: a minimalist approach to endoscopy. Gastroenterology 2018;154:1993-2003.

16. Wani SB, Williams JL, Komanduri S, Muthusamy VR, Shaheen NJ. Over-utilization of repeat upper endoscopy in patients with non-dysplastic Barrett's esophagus: a population-based study using a national benchmarking registry. Gastroenterology 2018;154(6 Suppl 1):S28-S29.

17. Tavakkoli A, Appelman HD, Beer DG, et al. Use of appropriate surveillance for patients with nondysplastic Barrett's esophagus. Clin Gastroenterol Hepatol 2018;16:862-869.e3.

18. Desai TK, Krishnan K, Samala N, et al. The incidence of oesophageal adenocarcinoma in non-dysplastic Barrett's oesophagus: a meta-analysis. Gut 2012;61:970-976.

19. Abrams JA, Kapel RC, Lindberg GM, et al. Adherence to biopsy guidelines for Barrett's esophagus surveillance in the community setting in the United States. Clin Gastroenterol Hepatol 2009;7:736-742; quiz 710.

20. Westerveld D, Khullar V, Mramba L, et al. Adherence to quality indicators and surveillance guidelines in the management of Barrett's esophagus: a retrospective analysis. Endosc Int Open 2018;6:E300-E307.

21. Wani SB, Williams JL, Komanduri S, Muthusamy VR, Shaheen NJ. Endoscopists biopsy the least those who need it the most: an analysis of Barrett's esophagus biopsy practices from a national quality benchmarking registry. Gastrointest Endosc 2018;87(6 Suppl):AB114.

22. Wani SB, William JL, Komanduri S, Muthusamy VR, Shaheen NJ. Regional variations and trends in quality indicator (QI) performance in Barrett's esophagus (BE): results from a population-based study using a national benchmarking registry. Gastrointest Endosc 2018;87(6 Suppl):AB537.

23. Curvers WL, ten Kate FJ, Krishnadath KK, et al. Low-grade dysplasia in Barrett's esophagus: overdiagnosed and underestimated. Am J Gastroenterol 2010;105:1523-1530.

24. Qumseya BJ, Wani S, Gendy S, Harnke B, Bergman JJ, Wolfsen H. Disease progression in Barrett's low-grade dysplasia with radiofrequency ablation compared with surveillance: systematic review and meta-analysis. Am J Gastroenterol 2017;112:849-865.

25. Schölvinck DW, van der Meulen K, Bergman JJGHM, Weusten BLAM. Detection of lesions in dysplastic Barrett's esophagus by community and expert endoscopists. Endoscopy 2017;49:113-120.

26. Moss A, Bourke MJ, Hourigan LF, et al. Endoscopic resection for Barrett's high-grade dysplasia and early esophageal adenocarcinoma: an essential staging procedure with long-term therapeutic benefit. Am J Gastroenterol 2010;105:1276-1283

27. Wani S, Mathur SC, Curvers WL, et al. Greater interobserver agreement by endoscopic mucosal resection than biopsy samples in Barrett's dysplasia. Clin Gastroenterol Hepatol 2010;8:783-788.

28. Standards of Practice Committee, Wani S, Qumseya B, et al. Endoscopic eradication therapy for patients with Barrett's esophagus-associated dysplasia and intramucosal cancer. Gastrointest Endosc 2018;87:907-931.e9.

29. Pech O, Behrens A, May A, et al. Long-term results and risk factor analysis for recurrence after curative endoscopic therapy in 349 patients with high-grade intraepithelial neoplasia and mucosal adenocarcinoma in Barrett's oesophagus. Gut 2008;57:1200-1206.

30. Shaheen NJ, Sharma P, Overholt BF, et al. Radiofrequency ablation in Barrett's esophagus with dysplasia. N Engl J Med 2009;360:2277-2288.

31. Phoa KN, van Vilsteren FG, Weusten BL, et al. Radiofrequency ablation vs endoscopic surveillance for patients with Barrett esophagus and lowgrade dysplasia: a randomized clinical trial. JAMA 2014;311:1209-1217.

32. Cotton CC, Haidry R, Thrift AP, Lovat L, Shaheen NJ. Development of evidence based surveillance intervals following radiofrequency ablation 
of Barrett's esophagus. Gastroenterology 2018 Apr 12 [Epub]. https://doi. org/10.1053/j.gastro.2018.04.011.

33. van Vilsteren FG, Alvarez Herrero L, Pouw RE, et al. Predictive factors for initial treatment response after circumferential radiofrequency ablation for Barrett's esophagus with early neoplasia: a prospective multicenter study. Endoscopy 2013;45:516-525.
34. Komanduri S, Kahrilas PJ, Krishnan K, et al. Recurrence of Barrett's esophagus is rare following endoscopic eradication therapy coupled with effective reflux control. Am J Gastroenterol 2017;112:556-566.

35. Qumseya BJ, Wani S, Desai M, et al. Adverse events after radiofrequency ablation in patients with Barrett's esophagus: a systematic review and meta-analysis. Clin Gastroenterol Hepatol 2016;14:1086-1095.e6. 